Supporting Information

\title{
Humidity-Driven Soft Actuator Built up Layer-by-Layer and Theoretical Insight into Its Mechanism of Energy Conversion
}

Huiyan Tan, Xiunan Yu, Yaqing Tu, Lidong Zhang*

School of Chemistry and Molecular Engineering, East China Normal University, Shanghai, 200241, People's Republic of China.

*E-mail: ldzhang@chem.ecnu.edu.cn

Keywords: layer-by-layer assembling, mathematic models, soft actuators, humidity, energy conversion

\section{Experimental Methods}

Materials: Sodium alginate $(\mathrm{SA}, \mathrm{Mw} \approx 50000)$, poly vinyl alcohol (PVA, $\mathrm{Mw} \approx$ 75000-79200), Dimethyl formamide (DMF), Agarose (AG) was purchased from commercial supplier (Sinopharm Chemical Reagent Co., Ltd). Polystyrene (PS, Mw $\approx$ 7400-9300) and polyvinylidene fluoride (PVDF, Mw $\approx 800000$, from Solvay, U.S.A) were purchased from Shanghai Titan Scientific Co., Ltd. Microscopy glass slides (50 
$\mathrm{mm} \times 50 \mathrm{~mm} \times 5 \mathrm{~mm}$, and $75 \mathrm{~mm} \times 25 \mathrm{~mm} \times 2 \mathrm{~mm}$ ) from T\&Q industries were washed with ethanol and water and dried at $50{ }^{\circ} \mathrm{C}$ before use.

Preparation of SA aqueous solution: SA powder $(5 \mathrm{~g})$ was dispersed in $175 \mathrm{~mL}$ deionized water in a $250 \mathrm{~mL}$ beaker and heated at $80{ }^{\circ} \mathrm{C}$ with vigorous stirring by a magnetic stir bar until complete dissolution of the powder. The final concentration of the solution was $28 \mathrm{mg} / \mathrm{mL}$. The solution was cooled down to room temperature and kept in a sealed container for the next use.

Preparation of PVA solution: PVA powder (3 g) was dispersed in $60 \mathrm{~mL}$ water in a $100 \mathrm{~mL}$ beaker and heated by microwave (Model: Midea microwave M1-L201B) to give the solution at the concentration of $50 \mathrm{mg} / \mathrm{mL}$. The solution was cooled down to room temperature and kept in a sealed container for the next use.

Preparation of SA@PVA composite solution: $20 \mathrm{~mL}$ glass bottles were marked as the number of (1), (2), (3), (4), (5), respectively. To each the bottle, $10 \mathrm{~mL} \mathrm{SA}$ solution was added by using a $12 \mathrm{~mL}$ syringe, followed by injecting the $1 \mathrm{~mL}$ PVA solution into bottle (1), $2 \mathrm{~mL}$ into bottle (2), $3 \mathrm{~mL}$ into bottle (3), $4 \mathrm{~mL}$ into the bottle (4), and $5 \mathrm{~mL}$ into the bottle (5). All bottles containing the SA@PVA composite solution were kept on a magnetic stirrer and heated at $90{ }^{\circ} \mathrm{C}$ with magnetic stirring for $2 \mathrm{~h}$. Various composite solutions were then cooled down to room temperature prior to the use for preparation of the film. The weight ratios of SA to PVA for final composite solutions were 5.6 (AP1 solution), 2.8 (AP2 solution), 1.9 (AP3 solution), 1.4 (AP4 solution) and 1.1 (AP5 solution) respectively. 
Preparation of AG solution: AG powder (1.8 g) was batchwise added into $50 \mathrm{~mL}$ DMF in a $100 \mathrm{~mL}$ beaker under the vigorous magnetic stirring. The mixture was heated at $90{ }^{\circ} \mathrm{C}$ for $4 \mathrm{~h}$ to ensure that all $\mathrm{AG}$ particles have been completely dissolved. The solution was cooled down to room temperature and kept in a sealed container for the next use.

Preparation of PS solution: PS particles $(0.2 \mathrm{~g})$ were dissolved in $50 \mathrm{~mL}$ DMF by magnetic stirring at $90{ }^{\circ} \mathrm{C}$ for $2 \mathrm{~h}$. The solution was cooled down to room temperature and kept in a sealed container for the next use.

Preparation of SA@PVA composite laminated film: The SA@PVA composite solutions from AP1 to AP5 were assembled into laminated films through layer-by-layer coating over a pre-cleaned glass template. The AP1 solution was coated on the glass template to give the first layer (Film-1). After drying at $40{ }^{\circ} \mathrm{C}$ in a natural convection oven, the first layer was treated by oxygen plasma for $10 \mathrm{~s}$, and coated with the AP2 solution to form the second bilayer (Film-2). These processes were repeated until forming the fifth layer to generate the laminated Film-5. The final thickness for all films (Film-1 to Film-5) was kept at 30 40 $\mu \mathrm{m}$.

Preparation of PVDF solution: To a 250-mL beaker filled with $80 \mathrm{~mL}$ DMF, PVDF powder $(5 \mathrm{~g})$ was added and heated at $90{ }^{\circ} \mathrm{C}$ accompanied by the vigorous magnetic stirring until complete dissolution of PVDF powder. The solution was then cooled to room temperature and kept in a static environment for the standby application. The final concentration of PVDF was $\sim 62 \mathrm{mg} / \mathrm{mL}$. 
Preparation of bilayers of SA/PVDF and PVA/PVDF: Two pre-cleaned glass templates $(12 \mathrm{~cm} \times 2 \mathrm{~cm} \times 2 \mathrm{~mm})$ were separately coated with $1 \mathrm{~mL}$ PVDF solution. The two samples were then moved to vacuum oven and heated at $80{ }^{\circ} \mathrm{C}$ for $2 \mathrm{~h}$ to give rise to uniform-thickness PVDF single layers. The two layers were treated with oxygen plasma for $30 \mathrm{~s}$, and one was coated with $1 \mathrm{~mL}$ SA solution, while the other one was coated with $0.5 \mathrm{~mL}$ PVA solution. After drying in air, the final SA and PVA layers reached the same thickness at $\sim 20 \mu \mathrm{m}$. The total thickness of the bilayer of SA/PVDF was similar to that of PVA/PVDF, so that the thickness effects on their bending motions could be weakened.

Tensile tests: The mechanical properties of all films were examined with a tensile tester (Model: HY-0580, Shanghai hengyi precise instrument limited company). A load cell of $50 \mathrm{~N}$ was used. The length was $30 \mathrm{~mm}$ for all the film samples. The samples were stretched at a speed of $20 \mathrm{~mm} / \mathrm{min}$.

Humidity-driven motions of various film strips: The film strip was kept on a support, and exposed to external humidity. All the motion processes were recorded through a digital camera (JVC, GC-PX100BAC). The bending angles and tip deflection were analyzed through comm software, ImageJ v.1.8.0 and HSSCLink. 


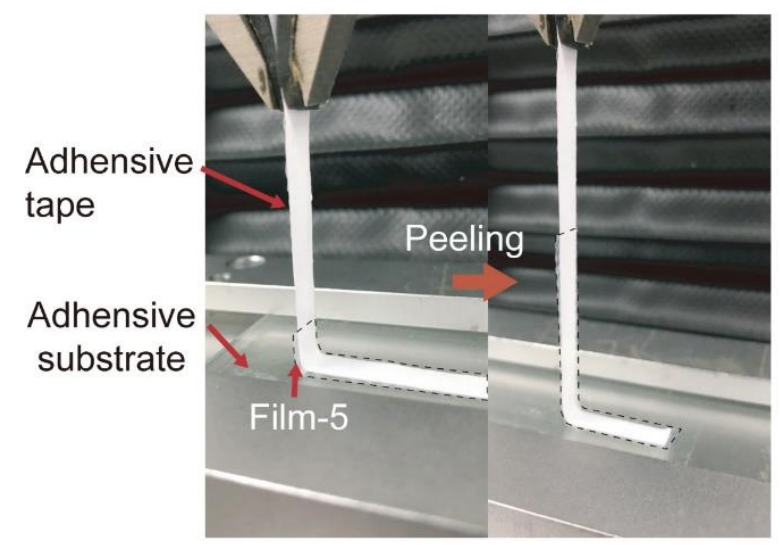

Figure S1. A standard $90^{\circ}$ peeling test. A multilayer Film-5 was fixed between a sticky substrate and a strip of non-elastic adhesive tape. Upon peeling at a rate of 10 $\mathrm{mm} / \mathrm{min}$, the Film-5 was separated from the substrate with still keeping its integrity, showing a strong interfacial interaction.


Figure S2. Mechanical tests of various multilayer films. (a) Three-point bending tests of SA and PVA single layers. (b) Stress-strain profiles of SA and PVA single layers. (c) Three-point bending tests of various multilayer films from Film-1 to Film-5. (d) Stress-strain profiles of Film-1 to Film-5. 

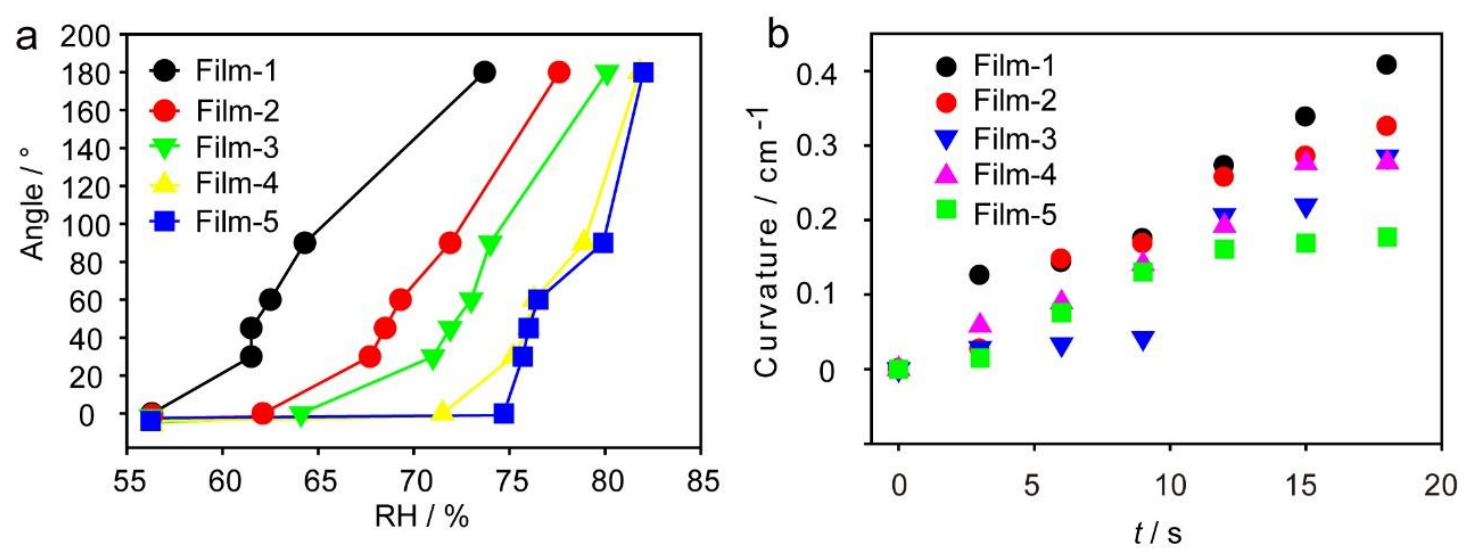

Figure S3. Humidity-driven bending tests. (a) The relative-humdity-dependence of the bending of various multilayer films. The relative humidity $(\mathrm{RH})$ for inducing the initial bending was 56\% for Film-1, 62\% for Film-2, 64\% for Film-3, 72\% for Film-4, and $75 \%$ for Film-5, and all films were capable of curling with reaching the same bending angles. (b) Curvature variation with time. By exposure to $\mathrm{RH}$ of $90 \%$, all films could bend immediately, and their bending processes in initial $18 \mathrm{~s}$ were recorded and analyzed by calculating the curvatures. Their curvatures at $18 \mathrm{~s}$ changed as an order of Film- $1>$ Film-2 > Film-3 $\geqslant$ Film-4 > Film-5. 


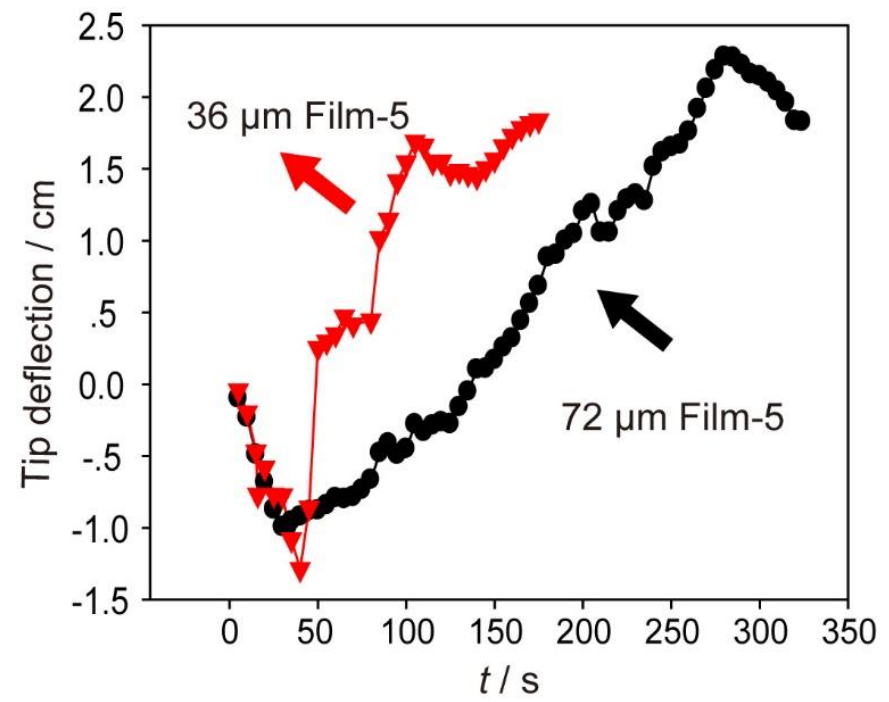

Figure S4. Tip deflection of thickness-different Film-5 strips driven by humidity.

The tip deflection-time profiles demonstrated a point of inflection during the curling that appeared at $\sim 40 \mathrm{~s}$. This point of inflection was still existed when the thickness was reduced to $36 \mu \mathrm{m}$. Such a tip deflection-time variation also reflected the non-uniform sensitivity and swellability along the thickness of the multilayer film of SA@PVA composites.



Figure S5. Bending cycling capability of Film-5 strip. Its bending was recorded and analyzed by a common software (HSSC Link1.3.4.34) by tracking its tip deflection, which indicated that a bending cycle required $\sim 50 \mathrm{~s}$, and this process was capable of proceeding for many cycles with still keeping its responsiveness to humidity. 

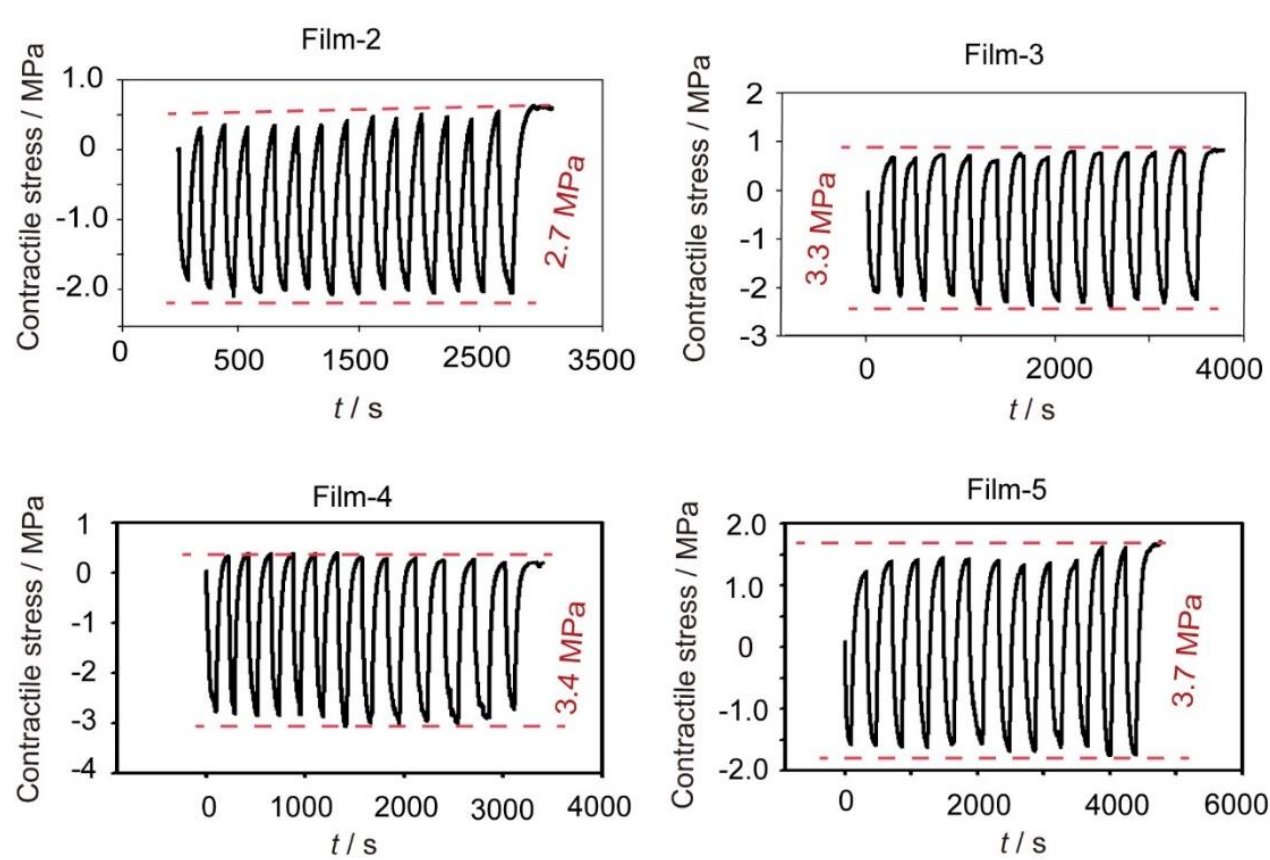

Figure S6. Contractile stress-time profiles of various films. This reversible bending generated the reversible variation of contractile stress that was tested on a tensile tester. The Film-5 strip ( $2 \mathrm{~cm} \times 2 \mathrm{~mm} \times 80 \square \mathrm{m})$ was clapped between two sample holders and kept straight by a pre-load of $0.02 \mathrm{~N}$. The gauge length was constant during the whole testing process. Upon being approached by water-immersed cotton, the film adsorbed humidity and expanded to generate a negative contractile stress. As the cotton was removed away, the film dehydrated with giving a positive contractile stress. The contractile stress increased with an increase in the PVA content ratio in the composite multilayers from Film-2 to Film-5. 


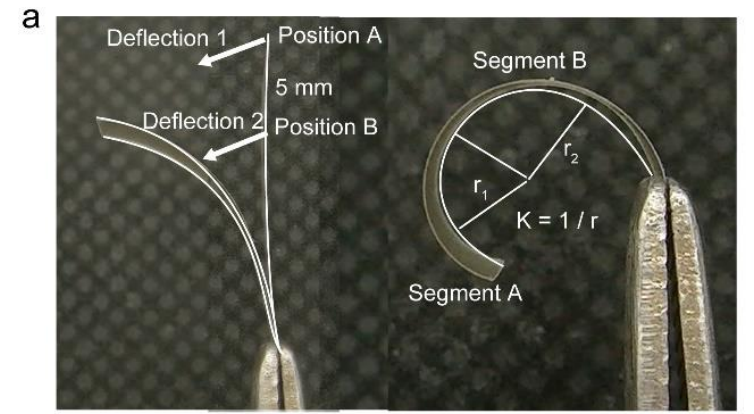

Film -5 with uniform thickness along its long axis

C

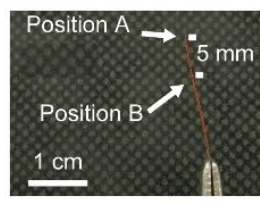

d



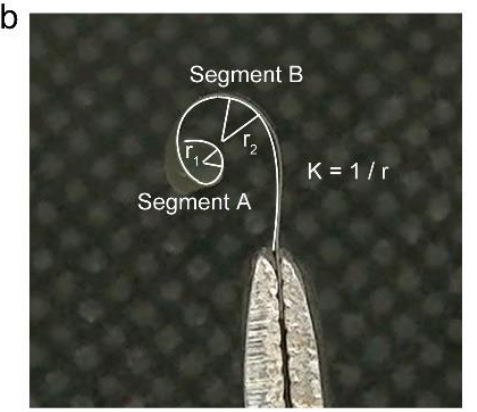

Film- 5 with non-uniform thickness along its long axis

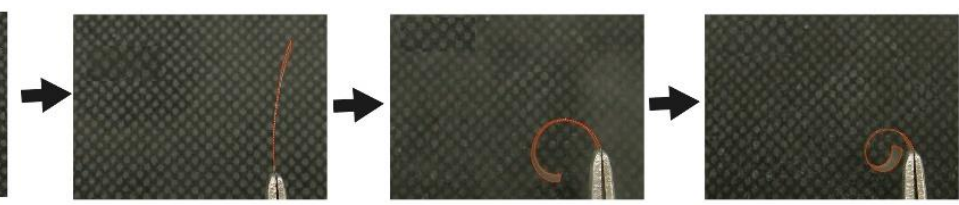

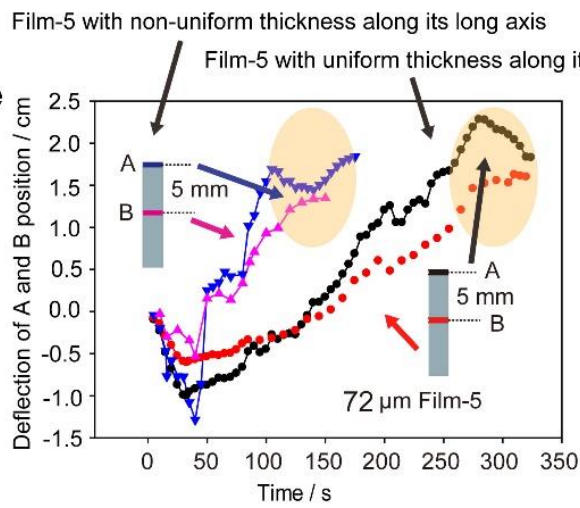

Film-5 with non-uniform thickness along its long axis

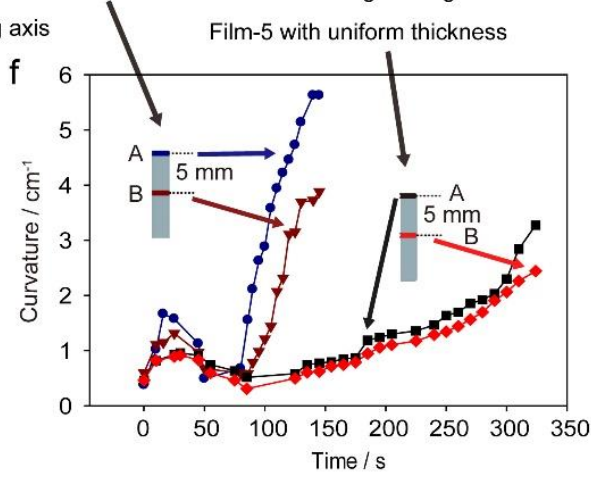

Figure S7. Humidity-driven curling motion of the Film-5 strips with uniform/non-uniform thickness. (a,b) Images showing the time-dependent deflection of the positions A and B for the non-/uniform-thickness strips of Film-5, where position A reflected the motion of the tip of strip and position B was $5 \mathrm{~mm}$ away from the A. (c) Snapshots of curling motion of the Film-5 strip with uniform thickness. (d) Snapshots of curling motion of the Film-5 strip with non-uniform thickness. (e) Time-dependent deflection of Film-5 strips with uniform/non-uniform 
thickness along their long axis. (f) Time-dependent curvatures of Film-5 strips with uniform/non-uniform thickness along their long axis.

Table S1. The correlation of relative humidity and bending degree of film actuators

\begin{tabular}{c|c|c|c|c|c|c}
\hline $\begin{array}{c}\text { Angle (') } \\
\text { RH (\%) }\end{array}$ & 0 & 30 & 45 & 60 & 90 & $\begin{array}{c}18 \\
0\end{array}$ \\
\hline Film-1 & 56. & 61. & 61. & 62. & 64.3 & 73. \\
& 3 & 5 & 5 & 5 & & 7 \\
\hline Film-2 & 62. & 67. & 68. & 69. & 71.9 & 77. \\
& 1 & 7 & 5 & 3 & & 6 \\
\hline Film-3 & 64. & 71 & 71. & 73 & 74. & 80. \\
& 1 & .0 & 9 & .0 & & 1 \\
& & & & & 0 & \\
\hline Film-4 & 71. & 75. & 76 & 76. & 78.9 & 81. \\
& 5 & 2 & .0 & 3 & & 8 \\
& 74. & 75. & 76 & 76. & 78.9 & 82 \\
\hline Film-5 & 7 & 7 & .0 & 5 & & .0 \\
\hline & & & & & & \\
\hline
\end{tabular}

\section{Legends of supplimentary Movies}

\section{Movie S1. Humidity-driven bending motion of a uniform-thickness strip of}

Film-5. An $80 \mu \mathrm{m}$ thick Film-5 first bent to AP5 side and then curled back into a uniform circle with continual sorption of humidity. The video is at its ten times speed.

Movie S2. Bending motion of various laminated films. By keeping the thickness constant, the ultimate curling curvatures varied as an order of Film-5 > Film-4 > Film-3 > Film-2 in response to the same gradient of humidity. The video is at its ten times speed. 
Movie S3. Comparison of the humidity-driven motility of SA/PVDF and

PVA/PVDF bilayers. These two simple models provided a visualized evidence; the higher the SA content, the higher the humidity sensitivity. The video is at its ten times speed.

Movie S4. Humidity-driven bending motion of a non-uniform-thickness strip of

Film-5. It also first bent to AP5 side and then returned with further curling into the maximum curvature toward AP1 side. However, its curling process was not uniform; the thinner segment curled more rapidly with the maximum curling curvature higher than the thicker segment, highly similar to that of Selaginella lepidophylla in response to gradient of humidity. The video is at its ten times speed.

Movie S5. Humidity-driven twisting motion of octopus-like shape of Film-5. The arms of the octopus first bent to the AP5 side and then bent back with further twisting toward AP1 side upon being approached with the water-saturated cotton.

Movie S6. Humidity-driven unfolding motion of bellflower-like shape of Film-5. The shape was initially closed with holding a small object by dehydration. As it approached to the water surface, the sorption of humidity resulted in its opening motion and releasing the small object out.

Movie S7. Humidity-driven bending motion of a dendritic shape cut from the non-uniform-thickness Film-5. The branching parts were thinner than the root segment. Driven by the sorption of humidity, the dendritic shape bent with grabbing a locomotive object. 